\title{
Effet de quatre souches de champignons mychoriziens arbusculaires sur Meloidogyne spp., principal nématode parasitaire du soja (Glycine max, L.) au Togo.
}

OGOU Anani ${ }^{1}$, TCHABI Atti2 ${ }^{\star}$, TOUNOU Agbéko Kodjo¹, AGBOKA Komi'1, SOKAME Bonoukpoè Mawuko ${ }^{3}$ ${ }^{1}$ Laboratoire de Recherche sur les Agroressources et la Santé Environnementale (LARASE) Équipe Défense des cultures ; École Supérieure d'Agronomie / Université de Lomé (ESA / UL).

2Laboratoire de Recherche sur les Agroressources et la Santé Environnementale (LARASE) Équipe Défense des cultures ; Institut Supérieur des Métiers de l'Agriculture, Université de Kara

${ }^{3}$ Team of Institute Research for Development (IRD) / International Centre of Insect Physiology and Ecology (ICIPE); Nairobi, Kenya

*Auteur correspondant : E-mail : attitchabi@yahoo.frTél : (+228) 91-78-13-81

Original submitted in on 12 th April 2018. Published online at www.m.elewa.org on $31^{\text {st }}$ July 2018 https://dx.doi.org/10.4314/jab.v127i1.1

\section{RÉSUMÉ}

Objectif: L'objectif de cette étude est d'évaluer l'effet de quatre souches de champignon mycorhizien arbusculaire d'origine locale sur la production du soja et le contrôle des nématodes parasitaires, Meloidogyne sp. au champ.

Méthodologie et résultats : L'essai a été conduit à la Station d'Expérimentation Agronomique de Lomé au Togo, de Juillet à Octobre 2016 suivant un dispositif de Fisher à quatre répétitions. Glomus mosseae, Glomus hoï, Acaulospora spinoza et BEN 10 ont été testées en comparaison au témoin. L'inoculation a été faite lors du semis du soja. II ressort de cette étude que les plants de soja inoculés sont fortement mycorhizés. Sur les quatre souches dans cette étude, Glomus mosseae a induit la meilleure réduction de la densité des nématodes dans les racines, entrainant une amélioration du rendement en graines du soja.

Conclusion et applications des résultats : Cette étude a montré que l'inoculation directe des CMA pendant le semis entraine une réduction de la densité des nématodes parasites et améliore le rendement du soja. Elle peut donc être un des moyens écologiquement durable de production du soja au Togo.

Mots clés : Inoculation mycorhizienne, nématodes, rendement, soja.

Effect of four inocula of arbuscular mycorrhizal fungi on Meloidogyne spp., main parasitic nematode of soybean (Glycine max, L.) in Togo.

\footnotetext{
ABSTRACT

Objective: The aim of this study is to assess the effect of four indigenous arbuscular mycorrhizal fungi inocula on Meloidogyne spp. population density and on the yield of soybeans in the field conditions.

Methods and results: The experiment was conducted at Experimental Station of Agronomy Faculty of University of Lomé in Togo, from July to October 2016, following a Fisher system with four replicates. Glomus mosseae, Glomus hoï, Acaulospora spinoza and BEN 10 were tested in comparison with the control. The results showed that the inoculated soybean plants were highly mycorrhized with higher number of nodules on the root.
} 
However, Glomus mosseae induced the highest reduction of Meloidogyne spp. density and damage in the roots, leading to improved soybean seed yield.

Conclusion and applications of the results: This study showed that direct inoculation of arbuscular mycorrhizal fungi at sowing period leads to Meloidogyne population density reduction and improving soybean grains yield. Using AMF can be a sustainable approach to produce soybean in Togo.

Keywords: Mycorrhizal inoculation, nematodes, yield, soybean.

\section{INTRODUCTION}

Le soja (Glycine max, L.) avec $35 \%$ de la production nationale, est la seconde légumineuse cultivée au Togo (DSID, 2014). La production du soja est estimée à 1650 tonnes en 2014 (FAOSTAT, 2016). II est considéré comme une culture de rente (DSID, 2014) et constitue ainsi un produit stratégique pour l'amélioration du revenu des producteurs (SCAPE, 2013). Le soja est l'une des légumineuses qui entre dans l'alimentation humaine et animale (Ogumniyi et al., 2012). Malgré ces atouts, le rendement du soja reste extrêmement très faible. II est en moyenne de 0,9 t/ha contre un rendement potentiel de 4t/ha (FAOSTAT, 2016). Ce faible rendement est dû aux problèmes de fertilités des sols et aux attaques des ravageurs notamment les nématodes. Les nématodes les plus dommageables à la culture du soja sont les nématodes à kystes du genre Heterodera dans les pays tempérés. Aussi les nématodes à galles du genre Meloïdogyne particulièrement dans les tropiques peuvent occasionner plus de $50 \%$ de perte de rendement (Wrather et al., 2003). Ces nématodes, en infestant les racines du soja rentrent en compétition pour les sites d'infections avec les bactéries fixatrices d'azote atmosphérique. Ce qui rend la plante vulnérable pour la formation des nodosités et donc affecte négativement la formation des gousses et des graines. Les traitements chimiques des sols à l'instar du Bromure de methyl, sont très efficaces pour lutter contre les nématodes (Johnson, 1985 ; Feldmesser

\section{MATÉRIELS ET MÉTHODES}

Site d'expérimentation: La présente étude a été réalisée à la Station d'Expérimentation Agronomique de l'École Supérieure d'Agronomie de l'Université de Lomé $\left(06^{\circ} 17^{\prime} \mathrm{N}, 001^{\circ} 21^{\prime} \mathrm{E}\right)$. Le climat est de type guinéen. II est caractérisé par deux saisons pluvieuses (une grande saison pluvieuse de mi-mars à mi-juillet et une petite saison sèche de septembre a mi-novembre). La et al., 1985 ; Giannakou et al., 2002 ; Brzeski et Coosemans, 2005). En revanche, leur utilisation est néfaste à la santé humaine ainsi qu'à l'environnement et ne représentent pas une solution idoine pour la promotion d'une agriculture durable (Brzeski et Coosemans, 2005).Les méthodes alternatives comme l'utilisation des extraits des plantes, la fumure organique et l'enfouissement des résidus des végétaux ainsi que la lutte biologique sont recommandées en raison des exigences de protection durable de l'environnement (Naseby et al., 2000). Cependant, I'utilisation des champignons mychoriziens arbusculaires (CMA) notamment les Glomeromycètes isolés localement pour une production durable du soja au Togo reste encore peu explorée (Smith and Read, 2008). Ces champignons sont reconnus très efficaces en cultures maraichères pour l'accroissement du rendement ainsi que pour le contrôle des nématodes (Affokpon et al., 2011, Bissadou et al., 2012; Osillos et Nagpala, 2014, Tchabi et al., 2016). Ce travail s'inscrit alors dans I'hypothèse que l'inoculation du soja avec les souches pures de CMA isolées localement pourraient permettre de réduire la densité de population des nématodes, leurs dégâts et ainsi qu'accroitre le rendement du soja. L'objectif donc de cette étude est d'évaluer l'effet de quatre souches pures de CMA sur le rendement du soja ainsi que sur la suppression des nématodes à galles

pluviométrie annuelle varie entre 800 et $1000 \mathrm{~mm}$. Le sol de type ferralitique, est qualifié de " terre de barre ». La composition chimique de sol au niveau de la station est de $1,87 \%$ de matière organique (MO), 0,15\% d'Azote (N) total, $0,05 \%$ de Phosphore $\left(\mathrm{P}_{2} \mathrm{O}_{5}\right) ; 0,46 \%$ de Potassium $\left(\mathrm{K}_{2} \mathrm{O}\right)$ et de $0,01 \%$ de Magnésium (MgO). L'analyse a été faite par le laboratoire de la Chimie du Sol 
de l'École Supérieure d'Agronomie, Université de Lomé. En début de l'essai, le site expérimental est naturellement infesté par des populations mixtes de nématodes à galles dominées par Meloidogyne sp. La densité moyenne d'infestation est de $87 \pm 12$ larves $\mathrm{J}_{2}$ pour $250 \mathrm{~cm}^{3}$ sol.

Dispositif expérimental et suivi de l'essai : Le dispositif utilisé est en blocs aléatoires complets à quatre répétitions afin de prendre en compte les variabilités spatiales (van Es et al., 2004). L'unité parcellaire a été de $15 \mathrm{~m}^{2}$. Le CMA à quatre niveaux : Le témoin (plants non inoculés), Glomus mosseae, Glomus hoi, Acaulospora spinoza et BEN 10 a été le seul facteur étudié. Le sol a été préparé une semaine avant le semis. Trois graines de soja ont été directement semées suivant le schéma cultural $60 \times 20 \mathrm{~cm}$ après ensemencement de $5 \mathrm{~g}$ d'inoculum de mycorhizes par poquet. Les poquets sont recouverts d'une couche fine de sable afin de favoriser la levée. A la levée, un démariage a été fait pour ne laisser que deux plants par poquet. II a été réalisé trois désherbages manuels avant la récolte pour éviter la concurrence nutritionnelle des adventices. La récolte a été faite 100 jours après semis quand les feuilles et les tiges ont commencé à sécher et que les gousses sont devenues brunes ou grises. Les graines ont été séchées après récolte afin de ramener le taux d'humidité à environs $12 \%$ pour une meilleure conservation.

Prélèvement et analyse des échantillons de sols et de racines : Un échantillon composite de $100 \mathrm{~g}$ de sol a été prélevé sur cinq points différents de chaque unité parcellaire suivant les diagonales avant le semis et à la récolte selon la méthode de Coyne et al. (2010). Ces cinq prélèvements ont été malaxés selon la méthode décrite par Barker et Niblack (1990) en utilisant un sachet plastique pour constituer l'échantillon représentatif d'une unité expérimentale. Chaque échantillon a servi à l'extraction et au dénombrement des nématodes dans le sol. Pour ce qui est des racines, un échantillon de $5 \mathrm{~g}$ de racines a été prélevé sur cinq plants choisis suivant les diagonales de chaque unité parcellaire. L'extraction et le dénombrement des nématodes des échantillons du sol et de racines ont été réalisés au Laboratoire de Virologies et de Biotechnologies Végétales / Unité d'Entomologie et de Nématologie (LVBV) de l'École Supérieure d'Agronomie de l'Université de Lomé (ESA / UL). Pour l'extraction des nématodes, la méthode de Bearmann Pan (Coyne et al., 2010) a été utilisée. Pour ce qui est des racines, elles ont été préalablement nettoyées à l'eau de robinet. Elles ont été ensuite découpées à l'aide d'un couteau en petits morceaux d'un centimètre. Chaque échantillon est mis dans un tamis tapissé à l'intérieur par du papier hygiénique qui joue le rôle de filtre. L'ensemble est placé dans une bassine en plastique (de $12 \mathrm{~cm}$ de diamètre et $25 \mathrm{~cm}$ de hauteur). Chaque échantillon est bien éparpillé dans le tamis en utilisant une pincette. Ensuite l'eau a été ajoutée jusqu'à couvrir légèrement l'échantillon de sols ou de racines, favorisant ainsi la migration des nématodes de l'échantillon vers l'eau qui constitue le milieu d'extraction. Après 24 heures et 48 heures respectivement pour les préparations de sol et de racines, le tamis est retiré délicatement de la bassine. L'eau de la bassine contenant les nématodes est recueillie dans une éprouvette graduée. Après 30 minutes de décantation, le volume de l'extrait a été ramené à $100 \mathrm{ml}$ constituant ainsi l'extrait qui servira à l'observation des nématodes. A cet effet, $10 \mathrm{ml}$ de l'extrait ont été prélevés à l'aide d'une pipette dans une boîte de Pétri quadrillée. Les nématodes sont observés à l'aide d'une loupe binoculaire de marque Olympus SZ12 à la magnification x 90. Quatre répétions de l'observation ont été faites.

\section{Évaluation du taux de mycorhization des racines :}

Pour l'évaluation du taux de mycorhization, les racines des jeunes plants ont été prélevées deux mois après semis (ou inoculation). Cinq plants ont été arrachés par unité parcellaire. L'échantillonnage a été fait de façon aléatoire. Ainsi Chaque échantillon est ramené au Laboratoire, bien lavé avec de l'eau de robinet ; puis $5 \mathrm{~g}$ de chaque échantillon a été constitué pour les observations microscopiques. Ces fragments de racines ont été colorés selon la méthode de Philippe et Hayman (1970). Les racines excisées ont été rincées à l'eau de robinet afin d'éliminer les particules de sables, d'argiles et/ou de limons. Elles ont été ensuite trempées dans de l'alcool à $90{ }^{\circ} \mathrm{C}$ pendant $24 \mathrm{~h}$ pour éliminé les microorganismes pouvant interagir avec les mycorhizes. Par ailleurs les racines prélevées ne doivent être ni trop jeunes, ni trop vieilles, mais peu ramifiées pour faciliter l'observation au microscope. Elles sont ensuite placées dans des tubes à essai contenant du KOH à $10 \%$. Ce traitement permet de décolorer les racines et de vider le contenu du cytoplasme des cellules racinaires. Les tubes sont placés au bain-marie à $90^{\circ} \mathrm{C}$ ependant $30 \mathrm{~min}$. Les racines sont par la suite rincées plusieurs fois à l'eau et colorées au bleu de trypan $(0,05 \%)$ pendant $30 \mathrm{~min}$. Les fragments de racines $(0,5 \mathrm{~cm})$ ont été déposés sur des lames avec quelques gouttes de glycérol. L'examen microscopique a été effectué à l'aide d'un microscope optique de marque Olympus ZX31 à la magnification $\mathrm{x}$ 90. Le taux de mycorhization a été calculé suivant la formule de Diouf et al. (2013) :

$T M(\%)=\frac{N R C}{N R} \times 100$ 
Où $\mathrm{TM}=$ Taux de mycorhization; NRC = Nombre de racines colonisées; $\mathrm{NR}=$ Nombre total de racines observées.

Paramètres agronomiques mesurés et calculés : La mensure de la croissance en hauteur des plants de soja a été faite chaque deux semaine sur dix plants choisis au hasard sur chaque unité parcellaire. Elle a commencé le 30 jour après semis (JAS) jusqu'au 95è jour. La mesure à l'aide d'un mètre ruban de la hauteur des plants est réalisée entre le collet et la feuille terminale. Celle de la longueur des racines a été faite en considérant uniquement les cinq racines les plus longues sur le système radiculaire du plant à la récolte. Ainsi, dix plants ont été choisis de façon aléatoire par unité parcellaire. Les nodosités symbiotiques de bactéries fixatrices d'azote atmosphérique ont été comptées de façon exhaustive. Le dénombrement a été effectué sur toutes les racines de dix plants choisis au hasard sur chaque unité parcellaire à la récolte. En effet, la distinction des nodosités symbiotiques sur les racines par rapport aux galles causées par Meloïdogyne est faite sur la base de leurs adhérences sur les racines. Ainsi, les nodosités symbiotiques adhèrent fortement aux racines et s'arrachent difficilement alors que les galles sont faiblement rattachées aux racines et tombent très facilement lorsque le plant de soja est secoué. L'évaluation de la masse de mille graines a été faite selon la méthode de I'ISTA (Edah, 2010) calculée avec la formule suivante :

$$
P(g)=\frac{\sum M L}{N L} \times 10
$$

\section{RÉSULTATS}

Taux de mycorhization des racines. Les résultats $d u$ taux de mycorhization des racines sont présentés sur la Figure 1. L'analyse de variances a révélé une différence significative entre les différents traitements $(F=11,77$; $d l=4 ; P=0,0004)$. La discrimination des moyennes par le test de Turkey au seuil de $5 \%$ a révélé que $G$.
Où $\Sigma M L=$ Somme des poids de chaque lot de 100 graines ; NL $=$ Nombre total de lots. L'estimation du rendement a été faite en pesant les poids totaux des graines récoltées sur chaque unité parcellaire de $15 \mathrm{~m}^{2}$ pour chaque traitement, puis ramenées en $\mathrm{Kg} / \mathrm{ha}$.

Analyse statistique des données : Toutes les données collectées ont été traitées avec le tableur Excel 2013 puis analysées avec le logiciel SPSS 20.0. Les comparaisons statistiques entre les différents niveaux de facteur ont été réalisées à l'aide d'une analyse de variance (ANOVA). Lorsque l'analyse révèle des différences significatives, le test de séparation des moyennes de Turkey (HSD) est appliqué au seuil de 5 $\%$. Les données relatives au nombre de gousses par plant, au nombre de nodosités symbiotiques de rhizobium formées par plant de soja et la densité des nématodes ont été transformées en $x^{\prime}=\log _{10}(x+1)$ avant l'analyse pour être normalisées, $x$ représentant les données brutes (Gomez et Gomez, 1984). Les données relatives au taux de mycorhization ont été transformées en $y^{\prime}=\arcsin \sqrt{y}$, y représentant les données en pourcentage (\%). Les données portant sur la hauteur moyenne des plants, la longueur moyenne des racines, la masse de 1000 graines et le rendement en graines du soja n'ont subies aucune transformation. Dans cette étude, le coefficient de corrélation de Pearson a été utilisé pour renseigner la dépendance de quelques variables étudiées. Par convention, la relation entre $X$ et $Y$ est : parfaite si $r=1$; très forte si $r>0,8$; forte si $r$ se situe entre 0,5 et 0,8 ; moyenne si r se situe entre 0,2 et 0,5 ; faible si $r$ se situe entre 0 et 0,2 et nulle si $r=0$ (Stern et al., 2006).

mosseae, $A$. spinoza et BEN 10 ont présenté des taux de colonisation significativement plus élevés $(63 \%, 59,9$ $2 \%$ et $65,67 \%$ respectivement) par rapport au Témoin $(28,89 \%)$ et à G. hoï (41 \%). G. hoïa induit un taux de colonisation de racines statistiquement identique aux plants témoins non inoculés. 


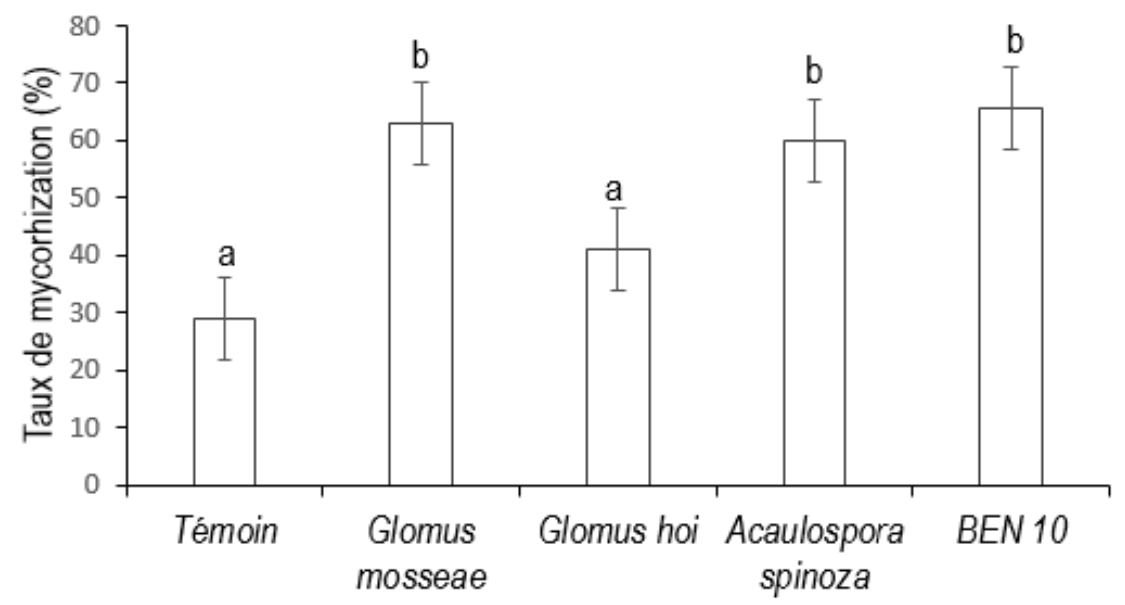

Souches de mycorhizes

Les barres portant les mêmes lettres sont statistiquement identiques à $P=0,05$ selon le test de Turkey (HSD).

Figure 1 : Taux de colonisation des racines de soja par quatre souches de mycorhizes

Effets de l'inoculation des CMA sur la longueur des racines des plants du soja : L'inoculation des plants de soja par les CMA a montré des effets statistiquement significatifs sur l'élongation des racines $(F=6,78 ; d l=$ 4; $P=0,004)$. La discrimination des longueurs moyennes par le test de Turkey au seuil de $5 \%$ a révélé que les plants inoculés par les CMA ont une longueur de racines moins importante que celle des plants témoins non inoculés. Cependant aucune différence significative n'est observée entre les différentes souches de CMA au seuil de $5 \%$ (Figure 2).

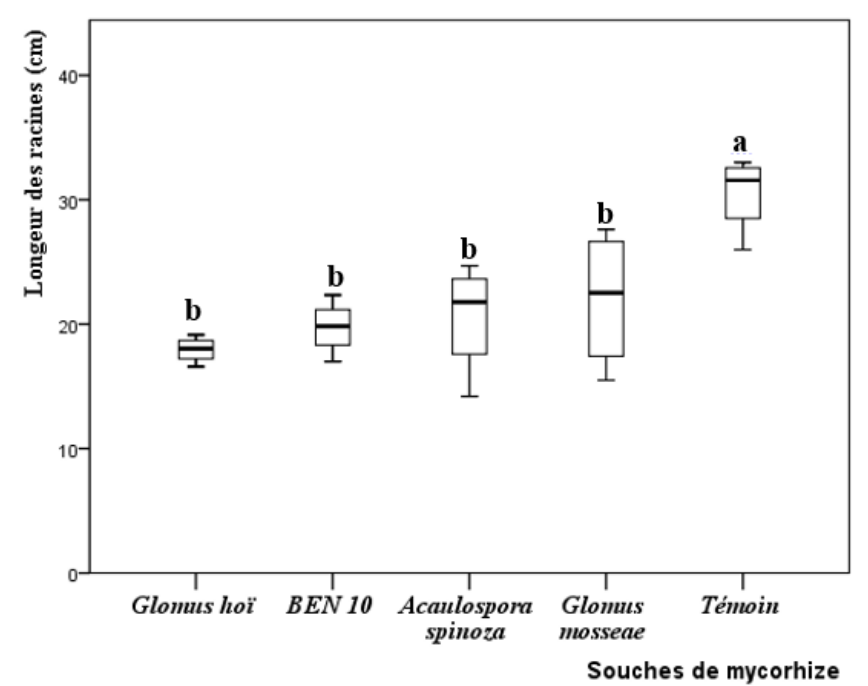

Les barres portant les mêmes lettres sont statistiquement identiques à $P=0,05$ selon le test de Turkey (HSD).

Figure 2: Effet de quatre souches de champignons mychoriziens arbusculaires sur la croissance en longueur des racines du soja

Effets de l'inoculation des CMA sur la hauteur moyenne des plants de soja : Les données relatives à l'effet de différents traitements sur la hauteur moyenne des plants à différentes dates du cycle végétatif de la culture sont présentées sur la Figure 3. L'ANOVA a révélé une différence significative entre les différents traitements au seuil de $5 \%(F=3,71 ; d l=4 ; P=$ 0,027 ) au $45^{\text {ème }}$ JAS. Les gains de croissance en hauteur sont respectivement de 16,93 $\pm 6,19$ pour $G$. mosseae, $14,05 \pm 7,38$ pour $G$. hoï, $32,03 \pm 9,03$ pour 
A. spinoza et de $14,26 \pm 4,42$ pour BEN 10 par rapport au témoin. L'infection mycorhizienne semble être effective à cette date, où il est observé une nette démarcation de hauteur de croissance entre les plants mycorhizés et ceux non mycorhizes. Par contre, aucune différence significative n'a été observée au 30ème $(F=$ $2,64 ; d l=4 ; P=0,07), 60$ ème $(F=0,45 ; d l=4 ; P=$ $0,77), 75^{\text {ème }}(F=1,72 ; d l=4 ; P=0,20)$ et $95^{\text {ème }}(F=$ 1,$09 ; d l=4 ; P=0,40$ ) (Figure 3) JAS, d'après l'ANOVA au seuil de $5 \%$.

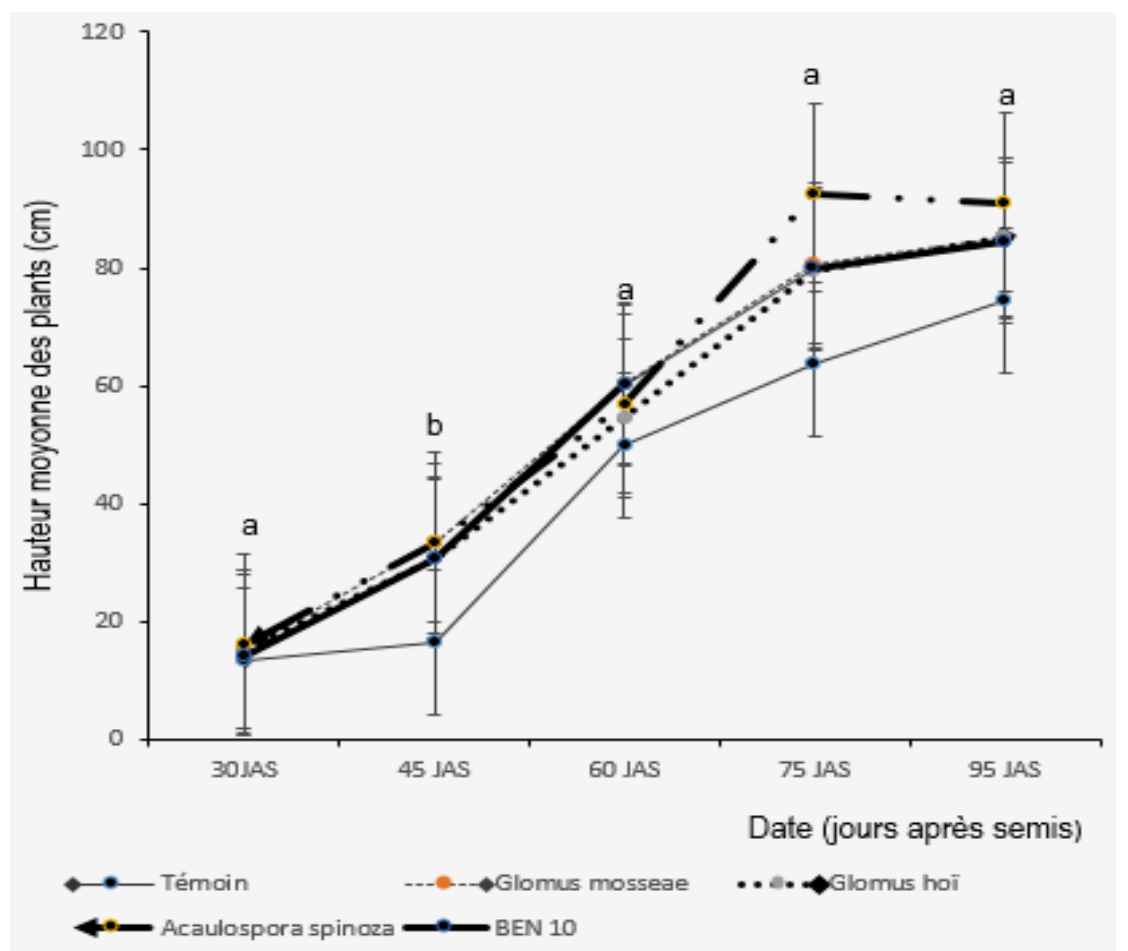

Les barres portant les mêmes lettres sont statistiquement identiques à $P=0,05$ selon le test de Turkey (HSD)

Figure 3 : Effet de quatre souches d'endomycorhizes sur la hauteur moyenne des plants de soja

Effets des différentes souches de mycorhizes sur la densité des nématodes parasites du soja : Les effets des souches de CMA sur la densité de population des nématodes parasites au niveau du sol et dans les racines de soja à la récolte sont présentés dans le Tableau 1.

Tableau 1: Effet de quatre souches de Champignons mychoriziens arbusculaires sur la densité de population de nématodes parasites au niveau du sol et des racines de soja à la récolte

\begin{tabular}{l|c|c|c}
\hline \multirow{2}{*}{ Traitement } & \multicolumn{2}{|c|}{ Sol (Densité / $\mathbf{1 0 0} \mathbf{g}$ de sol $\mathbf{\pm ~ S D ^ { 1 } )}$} & Racines (densité / $\mathbf{5 g}$ de racines à la récolte $\mathbf{\pm}$ SD') \\
\cline { 2 - 3 } & Avant semis & A la récolte & \\
\hline Témoin & $7,58 \pm 0,57 \mathrm{a}$ & $55,11 \pm 3,00 \mathrm{ab}$ & $12,75 \pm 6,40 \mathrm{a}$ \\
Glomus mosseae & $4,75 \pm 0,63 \mathrm{a}$ & $37,08 \pm 4,82 \mathrm{a}$ & $2,00 \pm 1,15 \mathrm{~b}$ \\
Glomus hoï & $7,25 \pm 2,15 \mathrm{a}$ & $46,14 \pm 7,37 \mathrm{ab}$ & $4,25 \pm 2,63 \mathrm{~b}$ \\
Acaulospora Spinoza & $6,75 \pm 1,73 \mathrm{a}$ & $56,57 \pm 6,55 \mathrm{ab}$ & $3,00 \pm 1,41 \mathrm{~b}$ \\
BEN 10 & $7,03 \pm 2,39 \mathrm{a}$ & $47,89 \pm 15,05 \mathrm{ab}$ & $2,75 \pm 0,96 \mathrm{~b}$ \\
\hline $\boldsymbol{F}$ & 1,781 & 3,573 & 7,440 \\
$\boldsymbol{P}$ & 0,197 & $0,039^{*}$ & $0,003^{* *}$ \\
dl & 4 & 4 & 4 \\
\hline
\end{tabular}

Dans une même colonne, les moyennes affectées d'une même lettre sont statistiquement identiques au seuil de $5 \%$ selon le test de Turkey (HSD). SD1 = Déviation Standard ; ${ }^{*}$ significatif $(p<5 \%)$, ${ }^{* *}$ hautement significatif $(p<1 \%)$. 
Au niveau du sol, la seule différence significative est notée entre $G$. mosseae et $A$. spinoza. Comparées au témoin, aucune différence significative n'est révélée avec les souches de mycorhizes inoculées. Ce qui veut dire que les souches de mycorhize utilisées n'ont pas d'effets significatifs sur la réduction de la densité des nématodes dans le sol. Par contre, au niveau des racines, il a été noté une différence hautement significative entre les souches de mycorhizes et le témoin. Ce qui veut dire que G. mosseae, G. hoï, A. spinoza et BEN 10 ont réduit la densité des nématodes au niveau des racines.

Effets des différentes souches de mycorhizes sur le rendement et ses composantes : Pour ce qui est du rendement en graines du soja, seule G. mosseae au seuil de $5 \%$, a un rendement significativement plus élevé $(2094,10 \pm 139,61 \mathrm{~kg} / \mathrm{ha})$ que le témoin $(636,53 \pm 296,02$ $\mathrm{kg} / \mathrm{ha})(\mathrm{p}=0,04)$. Par contre toutes les souches de mycorhizes ont donné des rendements similaires (Tableau 2). En ce qui concerne la formation des gousses sèches (nombre de gousses par plant), l'ANOVA a montré que seule $G$. mosseae a induit un rendement en gousses significativement plus élevé $(197,48 \pm 31,25)$ que le témoin $(87,06 \pm 23,81)$. ( $P<0,0001)$ et a donné un rendement en gousses significativement supérieur par rapport aux souches de mycorhizes. Les résultats sur le dénombrement des nodules symbiotiques ont montré que l'inoculation des quatre souches de mycorhizes a permis d'obtenir un nombre de nodules symbiotiques significativement plus élevé $(25,98 \pm 4,19)$ par rapport au témoin $(4,00 \pm 2,16$ (Tableau 2) $(P=0,0003)$. Par ailleurs, le nombre de nodules par plant est plus élevé avec la souche de BEN 10 ; alors que G.mosseae, G. hoi et $A$. spinoza ont donné un nombre de nodules statistiquement identique. Pour la masse de mille graines, A. spinoza et $G$. mosseae ont donné des résultats statistiquement identiques mais significativement différents par rapport au Témoin. Par contre, l'ANOVA a montré qu'il n'y a pas de différence significative entre les souches de mycorhizes à l'exception d'A. spinoza, qui a donné un poids de mille graines statistiquement différent par rapport aux autres souches.

Tableau 2 : Effets de quatre souches de champignons mychoriziens arbusculaires sur la formation de nodosités symbiotiques, de gousses, sur la masse de 1000 graines et le rendement en graines du soja

\begin{tabular}{|c|c|c|c|c|}
\hline Traitement & $\begin{array}{c}\text { Rendement_graines } \pm \\
\mathrm{SD}^{1}(\mathrm{~kg} / \mathrm{ha})\end{array}$ & $\begin{array}{l}\text { Rendement_- } \\
\text { gousses sèches } \pm \\
\text { SD }^{1} \text { (nombre/plant) }\end{array}$ & $\begin{array}{c}\text { Nombre de } \\
\text { nodules } \\
\text { symbiotiques } \pm \\
\text { SD }^{1} \text { (nombre/plant) }\end{array}$ & $\begin{array}{l}\text { Masse de } 1000 \\
\text { graines } \pm \mathrm{SD}^{1}(\mathrm{~g})\end{array}$ \\
\hline Témoin & $636,53 \pm 296,02 a$ & $87,06 \pm 23,81 a$ & $4,00 \pm 2,16 a$ & $107,50 \pm 10,67 a$ \\
\hline G. mosseae & $2094,10 \pm 139,61 b$ & $197,48 \pm 31,25 c$ & $14,21 \pm 2,32 b$ & $130,55 \pm 8,53 b$ \\
\hline Glomus hoï & $1725,48 \pm 828,69 a b$ & $135,83 \pm 21,57 b$ & $12,17 \pm 2,19 b$ & $123,33 \pm 7,20 a b$ \\
\hline A. spinoza & $1153,25 \pm 353,84 a b$ & $108,00 \pm 20,91 a b$ & $13,64 \pm 4,14 b$ & $107,50 \pm 10,67 a$ \\
\hline BEN 10 & $1372,63 \pm 54,44 a b$ & $154,11 \pm 6,87 b$ & $25,98 \pm 4,19 c$ & $133,55 \pm 2,57 b$ \\
\hline$F$ & 3,61 & 12,02 & 30,24 & 9,82 \\
\hline$P$ & $0,04^{*}$ & $0,0004^{* *}$ & $0,0003^{* *}$ & $0,0009 * *$ \\
\hline dl & 4 & 4 & 4 & 4 \\
\hline
\end{tabular}

Dans une même colonne, les moyennes affectées d'une même lettre sont statistiquement identiques selon le test de Turkey (HSD). $S D^{1}=$ Déviation Standard, * significatif $(\mathrm{p}<5 \%),{ }^{* *}$ hautement significatif $(\mathrm{p}<1 \%)$.

Corrélations entre le taux de mycorhization et les paramètres déterminants du rendement: Les analyses de corrélations linéaires de Pearson au seuil de $5 \%$ entre le taux de mycorhization et la longueur des racines, le nombre de nodosités symbiotiques, la formation des gousses, la densité des nématodes, le rendement en grains et masse de 1000 grains sont présentées sur la figure 4 ci-dessous. 


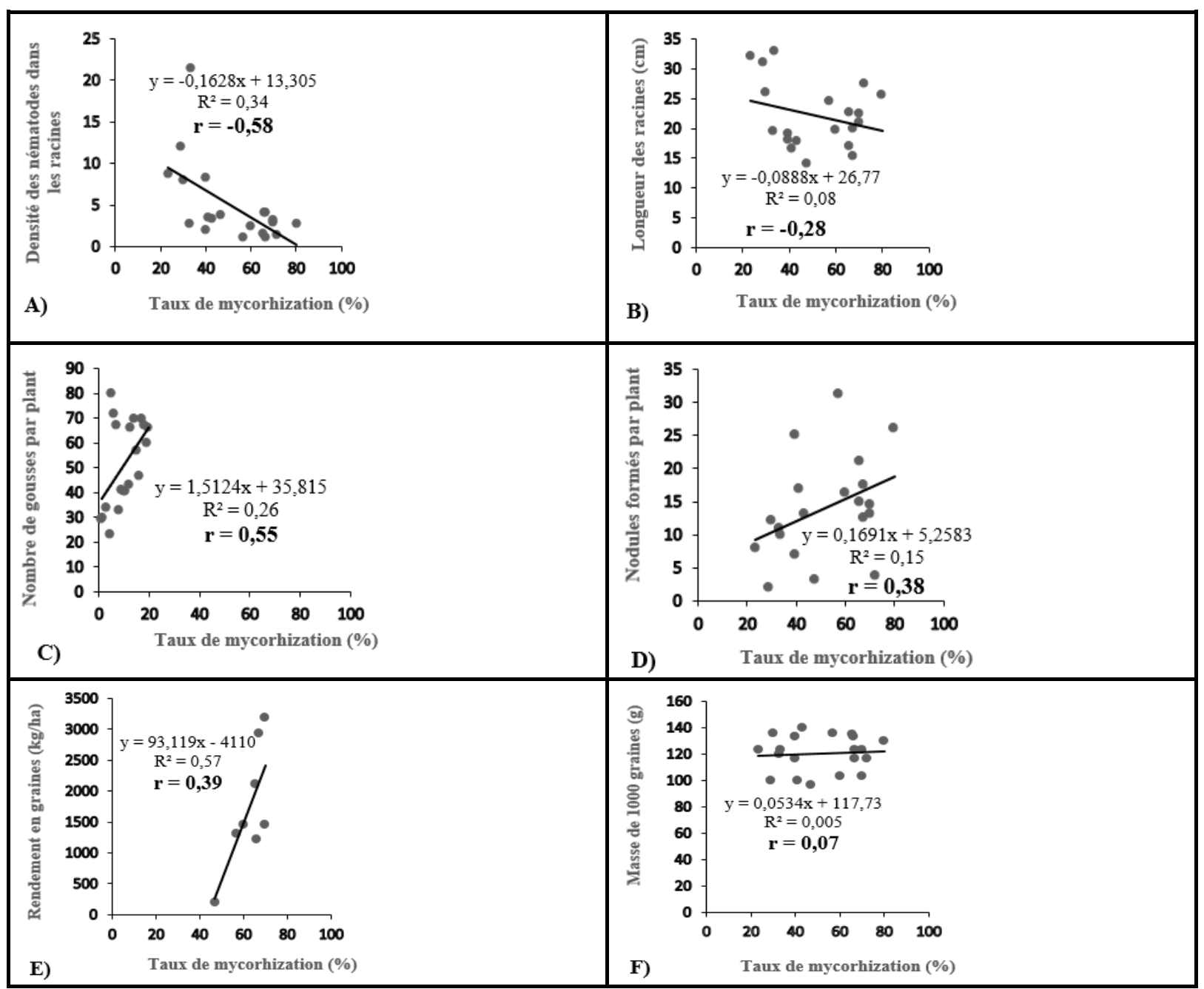

Figure 4 : Corrélations linéaires de Pearson entre les taux de mycorhization et quelques paramètres déterminants du rendement du soja

Les analyses de corrélations linéaires de Pearson ont montré (i) une corrélation significative négative et forte ( $\mathrm{r}$ $=-0,58 ; P=0,007$ ) entre le taux de mycorhization et la densité des nématodes dans les racines (figure 4A), (ii) une corrélation non significative négative et d'intensité moyenne $(r=-0,28 ; P=0.23$ ) entre le taux de mycorhization et la longueur des racines du soja (figure $4 B$ ), (iii)une corrélation significative positive et forte ( $r=$ 0,$55 ; P=0,013$ ) entre le taux de mycorhization et le nombre de gousses par plant (figure 4C), (iv) une

\section{DISCUSSION}

La colonisation des racines de soja par les champignons mycorhiziens arbusculaires a été observée aussi bien chez les plants inoculés que non inoculés. Chez les plants inocules, le taux de mycorhization des racines a été supérieur à $50 \%$ à l'exception de G. hoi. Ces corrélation non significative positive et d'intensité moyenne ( $r=0,38 ; P=0,09$ ) entre le taux de mycorhization et le nombre de nodules symbiotiques formés par plant (figure 4D), (v) une corrélation non significative positive et d'intensité moyenne $(r=0,39 ; P$ $=0.08$ ) entre le taux de mycorhization et le rendement en graines du soja (figure 4E), (vi) et enfin, une corrélation non significative positive et faible $(r=0,07$; $P=0,77$ ) entre le taux de mycorhization et la masse de 1000 graines (figure 4F) dans les conditions de l'étude.

résultats confirment plusieurs travaux antérieurs aux champs (Maggirwar et al., 2014 ; Berruti et al., 2016, Cely et al., 2016). Par contre, la mycorhization des plants non inoculés a été environ $28,89 \%$. Cette mycorhization des plants non inoculés est dû aux CMA indigènes actives 
que regorge le sol du site expérimentation. Par ailleurs, le faible taux de mycorhization enregistré au niveau des plants inoculés avec $G$. hoi s'expliquerait par plusieurs facteurs incluant la compatibilité des espèces ou souches de CMA avec la plante hôte (Öpik and Moora, 2012), leurs habitats, leurs niches écologiques et leurs compétitions vis-à-vis des Champignons natifs du sol (Verbruggen et al., 2013). Une augmentation du nombre de nodules de rhizobium au niveau des racines inoculées a été notée. Cette augmentation du nombre de nodules serait due à la stimulation des bactéries symbiotiques par les CMA inoculées. En outre, les CMA, en solubilisant le phosphore inaccessible au système racinaire du soja, rendent ce nutriment accessible aux racines, élément que les légumineuses exigent fortement pour leur croissance et surtout pour la nodulation (Gill et al., 1985 ). Des résultats similaires ont été obtenus sur la culture du niébé par N'Gbesso et al., (2013). Cependant, il est à noter qu'une bonne nodulation n'est pas toujours synonyme d'une bonne fixation d'azote car les nodules observés ne sont pas tous efficients (Onyibe et al., 2006). Concernant l'effet des CMA sur la croissance en hauteur des plants du soja, il ressort de nos résultats que l'inoculation des CMA n'a pas induit une amélioration de la croissance en hauteur des plants de soja à la fin du cycle végétatif. Ces résultats sont similaires à ceux obtenus par Cely et al., (2016), qui ont montré que les CMA appliqués seuls en champ, n'améliorent pas la croissance en hauteur de la culture du soja. Par contre, d'autre auteurs (Cheng et $T u, 1986)$ ont montré que Glomus clarum inoculé à la culture du soja à des densités de spores croissantes améliorent de façon significative la croissance en hauteur des plants de soja. Par ailleurs, les plants inoculés par les CMA ont une longueur de racines moins importante que celle des plants témoins non inoculés. Sidhoum (2011) a montré que la croissance en longueur serait souvent déviée au profit du développement de racines latérales. Cela pourrait s'expliquer par la présence de signaux moléculaires diffusibles dérivés du champignon qui stimulent la formation de racines latérales, occasionnant le développement du poids racinaire (Kosuta et al., 2003 ; Cely et al., 2016; Tchabi, 2016). Ainsi, les hyphes coloniseraient un grand volume de sol et le pénétrant à des profondeurs inaccessibles par les racines pour assurer la nutrition hydrominérale aux racines. D'autres études menées sur le plant d'olivier (Olea europea L.), effectués par Sidhoum (2011) ont montré que les plants inoculés présenteraient des longueurs de racines plus importantes que ceux non mycorhizés. Cela serait dû à la formation de glomalines qui favoriseraient une meilleure agrégation du sol et donc une meilleure pénétration des racines dans le sol (Porcel et al., 2003). Enfin, l'effet de l'inoculation des CMA sur le rendement a été évalué. L'évaluation est portée sur les gousses, les graines et le poids de 1000 graines. Ainsi, l'inoculation des plants de soja par les CMA a permis d'augmenter significativement le nombre de gousses formés au niveau des plants inoculés par rapport aux plants non inoculés. Au niveau de $G$. mosseae, le nombre de gousses par plant $(197,48 \pm 31,25)$ est plus élevé que celui du témoin $(87,06 \pm 23,81)$. Ce nombre important trouverait son explication dans la réussite de l'infection mycorhizienne, dû à la capacité des CMA à développer des hyphes et mobiliser l'eau et le phosphore soluble du sol (Smith \& Read, 2008). Ces résultats sont similaires à ceux de N'Gbesso et al., (2013) au Burkina Faso. Cet auteur avait constaté lors de son étude qu'une bonne mycorhization entraine une nodulation élevée chez le soja. Un bon remplissage des graines (masse de mille graines) serait fonction d'une bonne mycorhization et d'une bonne nodulation (Karaca et al., 2013 ; Taffouo et al., 2013; Mahanta et al., 2014 ; Xie et al., 2014). Malgré les conditions pluviométriques insuffisantes aux exigences du soja pendant la période de notre essai, il est noté un gain de rendement de la culture avec l'inoculation des CMA. Ce qui montre l'importance des endomycorhizes à permettre à la plante de lutter contre la sécheresse par l'amélioration de la nutrition hydrominérale (Smith \& Read, 2008). Concernant l'effet des CMA sur la densité des nématodes du soja, il a été noté une corrélation significative négative et forte entre le taux de mycorhization et la densité des nématodes dans les racines. En effet, plus les racines de soja sont mycorhizées, moins grande est la densité des nématodes, et vice-versa. Ceci s'expliquerait par la forte aptitude des CMA à réduire le taux de multiplication des nématodes par le développement des arbuscules dans les tissus racinaires (Hauggaard-Nielson, 2005). Ce développement constituerait des barrières physiques à la pénétration des nématodes (Smith \& Read, 2008). De plus l'amélioration du statut nutritionnel du soja ainsi que l'activation du système de défense de la plante confèrent une résistance des tissus racinaires vis-à-vis des attaques des nématodes (Smith \& Read, 2008). Le dénombrement des nématodes à la récolte a permis, d'enregistrer une forte augmentation de la densité de population de ceux-ci au niveau du sol. Les densités de A. spinoza, le Témoin, BEN 10, G. hoï et G. mosseae ont été de manière décroissante $49,90 \% ; 48,44 \% ; 41,22 \%$; $39,47 \%$ et $30,41 \%$ à la fin de la récolte du soja par rapport à la densité moyenne initiale de nématodes $(6,67$ 
$\pm 1,49$ ) dans le sol avant le semis. Cette forte augmentation de la population des nématodes s'expliquerait par: (i) le cycle de vie très court des nématodes parasites du soja, (ii) le stade de développement auquel étaient les nématodes dans le sol avant le semis, (iii) la présence de l'hôte (soja) qui sécrète des exsudats racinaires favorisant leur développement grâce à l'émission de signaux moléculaires racinaires qui préviennent de l'existence d'un hôte potentiel et par conséquent d'une source de carbone organique, (iv) les conditions favorables d'humidité, de $\mathrm{pH}$ du sol pour leur développement et au mode de labour (Cardoso \& Kuyper,, 2006), (vi) et l'humectation pour faciliter l'échantillonnage de sol et de

\section{CONCLUSION}

Cette étude a montré que l'inoculation directe du soja aux quatre souches de champignons mychoriziens arbusculaires au moment du semis a permis de réduire la densité de population de nématodes au niveau des racines et a entrainé une amélioration du rendement en graines. De toutes les souches de mycorhizes racine au moment de la récolte où le sol est sec et dur. Ce qui aurait favorisé la migration des nématodes à la surface du sol (Bissadu et al., 2012). Par contre, à la récolte, le nombre moyen des nématodes semble identique en dépit de l'inoculation mycorhizienne dans le sol. L'application des CMA seuls n'a pas permis une réduction de la densité des nématodes au niveau du sol dans les conditions de notre essai. Certaines substances toxiques peuvent être libérées lors de la sécrétion des exsudats racinaires de la culture, repoussant ainsi les nématodes, qui migreraient vers les couches profondes du sol. La susceptibilité de la culture mise en place pourrait également être un facteur explicatif de la réduction du taux d'infestation des nématodes.

inoculées, G. mosseae a induit la meilleure réduction des nématodes parasites au niveau des racines et a permis d'augmenter le rendement en graines du soja. Son utilisation par les producteurs serait donc écologiquement rentable.

\section{REMERCIEMENT}

Nous remercions l'Institut International d'Agriculture Tropical d'Abomey-Calavi / Benin (IITA) pour la multiplication au Laboratoire des souches de champignons mychoriziens arbusculaires.

\section{RÉFÉRENCES}

Affokpon A, Coyne DL, Htay CC, Agbèdè RD, Lawouin L, Coosemans J, 2011. Biocontrol potential of native Trichoderma isolates against root-knot nematodes in West African vegetable production systems. Soil Biology \& Biochemistry 43: 600-608.

Barker KR et Niblack, TL, 1990. Soil sampling methods and procedures for field diagnosis. Plant Nematology Laboratory Manual. Revised Edition. The University of Massachusetts Agricultural Experiment Station, Amherst, MA, USA. pp., 10-19.

Berruti A, Lumini E, Balestrini R, and Bianciotto V, 2016. Arbuscular Mycorrhizal Fungi as Natural Biofertilizers: Let's Benefit from Past Successes. Frontiers in Microbiology, 6:1559. https://doi.org/10.3389/fmicb.2015.01559.

Bissadou KD, Tchabi A, Tounou AK, Ayessom A, Gumedzoe M, 2012. Impact de la fumure organique et de champignon Glomus mosseae sur Meloidogyne spp en culture de tomate au Togo. Journal of Applied Biosciences 55: 39733986.
Brzeski MW et Coosemans J, 2005. Integrated pest management, in: Introduction to the biology and management of Nematodes Interacting with Agro Ecosystems. Fascicule for Postgraduate International Nematology Course. Université de Gand, 40p.

Cardoso I. M., and Kuyper T. W., 2006. Mycorrhizas and tropical soil fertility. Agric. Ecosyst. Environ., 116 : 72-84.

Cely MVT, d'Oliveira AG, de Freitas VF, de Luca MB, Barazetti AR, dos Santos IMO, Gonco B, Garcia GV, Prete CEC, Andrade G, 2016. Inoculant of Arbuscular Mycorrhizal Fungi (Rhizophagus clarus) Increase Yield of Soybean and Cotton under Field Conditions. Frontiers in Microbiology 7: 720. Doi: 10.3389/fmicb.2016.00720.

Cheng. YH et Tu CC, 1986. Effects of Vesiculararbuscular mycorrhiza on the absorption of Phosphorus. Growth and yield in corn and soybean. Tainan Dist. Agr. Impro. Sta. Bull. No. 20:39-52. 
Coyne DL, Nicol JM et Claudius-Cole B, 2010. Les nématodes des plantes : Un guide pratique de terrain des techniques de terrain et de laboratoire, traduit par Patrick Quénéhervé, Integrated Pest Management (IPM), 93p.

Diouf D, Fall D, Kane A, Bakhoum N, Ba AT, Ba AM, Duponnois R, 2013. Effet de l'inoculation avec des souches de mesorhizobium sp. et / ou des champignons mycorhiziens a arbuscules sur la croissance et la nutrition minérale de plants d'acacia. Seyal. ISBN : 978-2-7099-1827-5.

Direction des Statistiques Agricoles, de l'Informatique et de la Documentation (DSID), 2014. Principales caractéristiques de l'agriculture togolaise, 4ème recensement national de l'agriculture 2011-2014 / volume 4: module complémentaire ; $165 p$.

Edah K, 2010. Guide pour la production et le contrôle de semences certifiées, rapport de consultation du projet GPC/TOG/016/EC. Togo; 84p.

Feldmesser, J., Kochansky, J., Jaffe, H., and Chitwood, D., 1985. Future chemicals for control of nematodes, p.327-344, in: "Agricultural Chemicals of the future," J. L. Hilton, ed., Rowman and Allanheld, Totowa, New Jersey.

Food and Agriculture Organization Statistical database (FAO STAT), 2016. Les derniers enregistrements pour l'indicateur : Soja Production (Tonnes) : Togo. http I 20modiale\%202016/Togo\%20-\%20Soja\%20$\% 20$ Production $\% 20$ (Tonnes) $\% 20$ $\% 202016$.html.

Giannakou I. O., Sidiropoulos A. and ProphetouAthanasiadou D., 2002. Chemical alternatives to methyl bromide for the control of root-knot nematodes in greenhouses. Pest Management Science, 58:290-296. DOI : 10.1002/ps.453

Gill MA, Ali N., Nayyar MM, 1985. Relative effect of phosphorus combined with potash and Rhizobium phaseoli on the yield of Vigna aureus (mung). Journal of Agricultural Research, 23: 279-282.

Gomez A.K. et Gomez AA, 1984. Statistical procedures for agricultural research 2nd ed. Wiley et Sons, New York, USA.680 pp.

Hauggaard-Nielson, 2005. Intercropping of Wheat and Pea as Influenced by Nitrogen Fertilization. Nutrient Cycling in Agroecosystems., 73 (2): 201:201-21273. Doi: 10.1007/s10705-0052475-9.
Johnson AW, 1985. The role of nematicides in nematode management. In: Sasser J.N. and Carter C.C. (Eds.). An Advanced Treatise on Meloidogyne. Volume 1. Biology and Control. North Carolina University Graphics, Raleigh, NC, USA. Pp. 249-267.

Karaca H, Uygur V, Özkan A, Kaya Z, 2013. Effects of mycorrhizae and fertilization on soybean yield and nutrient uptake. Communications in Soil Science and Plant Analysis 44: 24592471.Doi:10.1080/00103624.2013. 809730.

Kosuta, S, Chabaud M, Lougnon G, Gough C, Denarie J, Barker D, Bécard G, 2003. A Diffusible Factor from Arbuscular Mycorrhizal Fungi Induces Symbiosis-Specific MtENOD11 Expression in Roots of Medicago truncatula. Plant Physiology, 131(3) :952-962. 3

Maggirwar RC, Parimal SS, Khodke SP and Deotare, PW. 2014. Arbuscular mycorrhizal and dark septate endophytic association in some grasses from Amravati (M.S.). International Journal of Development Research, 4(12)26112614.

Mahanta D, Rai RK, Mishra SD, Raja A, Purakayastha TJ, Varghese E, 2014. Influence of phosphorus and biofertilizers on soybean phosphorus fertilization, and intraspecific density. Mycorrhiza, 15: 203-221. Doi: 10.1007/s00572004-0324-3.

N'gbesso FDPM, Fondio L, Dibi Brice EK, Djidji H, Kouame A, N'gbessan C, 2013. Étude des composantes du rendement de six variétés améliorées de niébé [Vigna unguiculata (L.) Walp]. Journal of Applied Bioscience 63: 4754 $-4762$.

Naseby DC, Pascual JA, Lynch JM, 2000. Effect of biocontrol strains of Trichoderma on plant growth, Pythium ultimum population, soil microbial communities and soil enzyme activities. Journal of Applied Microbiology, 88:161-169.

Ogumniyi S, Johan W, Andries J, Roem W, 2012. Bacterial translocation and in vivo assessment of intestinal barrier permeability in rainbow trout (Oncorhynchus mykiss) 51 with and without soyabean meal-induced inflammation. Journal of Nutritional Science, (5): 1-10

Onyibe JE, Kamara AY, Omoigui LO, 2006. Guide to cowpea production in Borno State, Nigeria. Promoting Sustainable Agriculture in Borno State (PROSAB), Ibadan, Nigeria. 36 pp. 
Öpik M and Moora M, 2012. Missing nodes and links in mycorrhizal networks. New Phytologist 194: 304-306.doi:10.1111/j.14698137.2012.04121.x.

Osillos, PL, Nagpala AL, 2014. The Effects of Arbuscular Mycorrhizal Fungi (AMF) as biofertilizer on the growth, yield and nutrient uptake of tomato (Lycopersicon esculentum Mill.). International Journal of Scientific Engineering and Research, volume $3(11): 49-65$.

Phillips J M and Hayman DS, 1970. Improved procedures for clearing and staining parasitic and vesicular-arbuscular mycorrhizal fungi for rapid assessment of infection. Transactions of the British Mycological Society, 55:158-161.

Porcel R, Manuel J et Lozano R, 2003. Arbuscular mycorrhizal influence on leaf water potential, solute accumulation, and oxidative stress in soybean plants subjected to drought stress. Journal of Experiment Botany, 55 (403) : 17431750.

Sidhoum W, 2011. Diversité des mycorhizes arbusculaires chez la variété "Sigoise" d'olivier (Olea europea L.) : étude de leur efficacité sur la croissance des plants. Mémoire de Magister en Biotechnologie / Maroc, 91p.

Smith SE and Read DJ, 2008. Mycorrhizal symbiosis. Third Edition, London, UK, Academic press; $787 \mathrm{pp}$.

Stern R, Knock J, Rijks D, Dal I, 2006. Instat + for windows. Version 3.30. An interactive Statistics Package. Reading : University of Reading UK, 2006.

Stratégie nationale de croissance accélérée et de promotion de l'emploi (SCAPE), 2013. Document de Stratégie nationale de croissance accélérée et de promotion de l'emploi au Togo de 2013-2017. Version semi-définitive, 200p.

Taffouo VD, Ngwene B, Akoa A, Franken P, 2013. Influence of phosphorus application and arbuscular mycorrhizal inoculation on growth, foliar nitrogen mobilization, and phosphorus partitioning in cowpea plants. Mycorrhiza 24: 361-368.doi:10.1007/s00572-013-0544-5.

Tchabi A, Hountondji FCC, Ogunsola B, Lawouin L, Coyne D, Wiemken A, Oehl F, 2016. The Influence of Arbuscular Mycorrhizal Fungi Inoculation on Micro-Propagated Hybrid Yam (Dioscorea spp.) Growth and Root Knot Nematode (Meloidogyne spp.) Suppression. nt.Journal of Current Microbiology Applied
Science 5(10): 267-281. doi: http://dx.doi.org/10.20546/ijcmas.2016.510.030

Van Es HM, Gomes C, Sellmann M, van Es CL, 2004. Spatially- balanced designs for experiment's on autocorrected fields. In: 2004 Proc. Am. Statistical Assoc., Statistics \& the Environnement Section (CD ROM). Am. Statistical Assoc., Alexandria, VA.

Verbruggen $F$, Adams RC, van't Wout $F$, Stevens $T$, McLaren IPL, \& Chambers CD, 2013. Are the effects of response inhibition on gambling longlasting? PLOS ONE, 8(7), e70155. Doi: 10.1371/journal.pone.0070155.

Wrather JA, Koenning SR, Anderson TR, 2003. Effect of diseases on soybean yields in the United States and Ontario (1999-2002). Online. Plant Health Progress Doi: 10.1094/PHP-2003-032501-RV.

Xie X, Wenga BD, Cai B, Dong Y, Yan C, 2014. Effects of arbuscular mycorrhizal inoculation and phosphorus supply on the growth and nutrient uptake of Kandeliao bovata seedlings in autoclaved soil. Applied Soil Ecology, 75 : 162171.doi:10.1016/j.apsoil.2013. 11.009. 